\title{
Conceptual Design of Tunable Structures for Mid-Frequency Response
}

\author{
Ercan M. Dede and Gregory M. Hulbert* \\ Department of Mechanical Engineering, University of Michigan, Ann Arbor, MI, 48109-2125, U.S.A.
}

The conceptual design of tunable structures for mid-frequency dynamic response is discussed. The influence of changing the mechanical advantage of a structural unit cell is studied at multiple scales. Incorporation of adaptive materials into a unit cell is also examined. Results from these investigations link amplification properties and variable material parameters to tunable response with implications toward the development of unique shape adaptive composite structures for aerospace applications.

\section{Nomenclature}

$\begin{array}{ll}A & =\text { cross-sectional area } \\ E & =\text { Young's modulus } \\ E^{*} & =\text { complex Young's modulus } \\ \eta & =\text { loss factor } \\ \mathbf{F}_{\text {in }} & =\text { input force vector } \\ \mathbf{F}_{\text {out }} & =\text { output force vector } \\ I & =\text { area moment of inertia } \\ \lambda & =\text { wavelength of vibration } \\ p_{\text {in }} & =\text { incident pressure field amplitude } \\ P_{\text {in }} & =\text { input power } \\ P_{\text {out }} & =\text { output power } \\ r_{\text {in }} & =\text { input radius } \\ r_{\text {out }} & =\text { output radius } \\ \rho & =\text { density } \\ \mathbf{v}_{\text {in }} & =\text { input velocity vector } \\ \mathbf{v}_{\text {out }} & =\text { output velocity vector } \\ v_{\text {in }} & =\text { input velocity magnitude } \\ v_{\text {out }} & =\text { output velocity magnitude }\end{array}$

\section{Introduction}

Many passive methods for reducing structural vibrations exist ${ }^{1,2}$ including discrete damping devices, visco-elastic coatings, ${ }^{3}$ and absorbent padding. Additionally, periodic structures ${ }^{4,5}$ have been considered for vibration control, and one recent approach ${ }^{6}$ is to use small complaint mechanisms within large periodic structures for the reduction of mid-frequency (i.e. $1-10 \mathrm{kHz}$ ) vibratory response. This latter method is centered upon the selection of functional mechanism topologies that have a large mechanical advantage. Computational and experimental investigations ${ }^{7}$ have demonstrated the efficacy of this passive strategy while employing different unit cell designs.

To further extend this concept of structural control, presented herein are methods to establish connections between variable amplification properties, adaptive material properties, and tunable structural dynamic

*Professor, Department of Mechanical Engineering, University of Michigan, 2250 G.G. Brown, 2350 Hayward, Ann Arbor, MI 48109-2125. 
response. The study of these relationships is motivated by applications in which real-time control over the structural dynamics of a vehicle chassis is sought in response to a changing flight regime. Thus, these relationships are first explored by modifying the shape of an underlying integral compliant mechanism topology within a unit cell composed of a conventional material and then computing the resultant dynamic behavior of the unit cell and a corresponding full structure. Following this, a composite unit cell comprising shape memory and other alternative materials with actuators is examined for further enhancement of response tunability. Periodic sandwich panel structures with integral compliant mechanisms, actuators, and adaptive materials for control of structural dynamic response are then suggested as a logical extension of this work and as a possible embodiment of the types of systems suggested by Trease. ${ }^{8}$

In Sec. II a brief review is provided of a prototypic compliant mechanism unit cell that serves as a template for subsequent design studies. A concept for a new shape adaptive unit cell is then outlined in Sec. III. In Sec. IV the link between varying the mechanical advantage of the unit cell and mid-frequency dynamic response is established. The dynamic analysis of two related periodic structures is then presented in Sec. V to connect local and global level performance. Extensions towards shape adaptive composite structures with adaptive materials for greater structural control are provided in Sec. VI. A summary of this research is provided in Sec. VII.

\section{Brief Review of Concepts}

The rigid link mechanism shown in Fig. 1(a) is a straight-line displacement inverter, where the mechanism points of force input and output move in opposite directions. The corresponding compliant mechanism unit cell embodiment of this rigid link mechanism is shown in Fig. 1(b). ${ }^{7}$

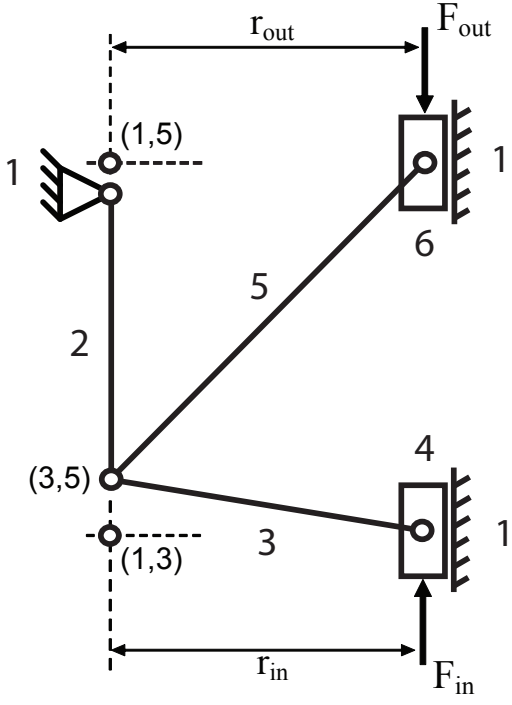

(a)

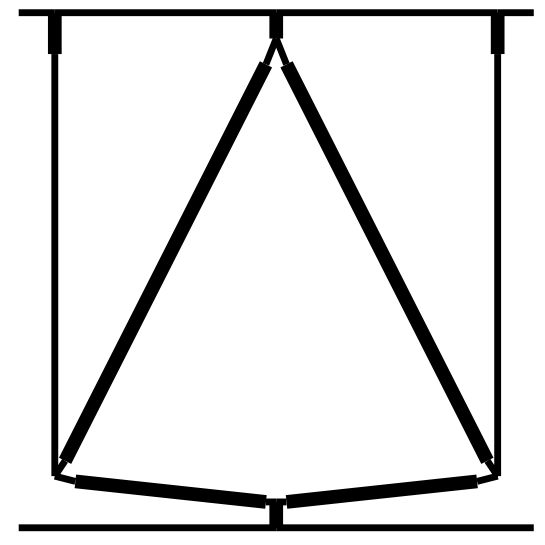

(b)

Figure 1. (a) Rigid link diagram of a rhombus mechanism (numbers denote links; (.,.) denote instant centers); (b) Rhombus compliant mechanism unit cell $(\mathrm{MA}=17)$.

This mechanism topology was selected since it has a large mechanical advantage. ${ }^{9}$ By constructing a unit cell in this manner and subjecting the bottom layer to a pressure load the top layer root-mean-square (RMS) velocity is reduced within predicted frequency ranges as a result of the unit cell amplification properties. Furthermore, this local behavior translates to reduced vibratory response of global compliant mechanism structures. ${ }^{6,7}$

\section{II.A. Mechanical Advantage Relationships}

The above concept is predicated upon a mechanical advantage analysis ${ }^{10}$ of a given mechanism topology. Taking the respective input and output force vectors as $\mathbf{F}_{\text {in }}$ and $\mathbf{F}_{\text {out }}$, and the respective velocity vectors of 
the points of force input and output as $\mathbf{v}_{\text {in }}$ and $\mathbf{v}_{\text {out }}$, and under the simplifying (but not limiting) assumption of non-dissipative mechanisms, gives:

$$
P_{\text {out }}=P_{\text {in }} \quad \Rightarrow \quad \mathbf{F}_{\text {out }}^{\mathrm{T}} \mathbf{v}_{\text {out }}=\mathbf{F}_{\text {in }}^{\mathrm{T}} \mathbf{v}_{\text {in }},
$$

where $P_{\text {out }}$ is the mechanism output power and $P_{\text {in }}$ is the mechanism input power.

By extending this analysis to the rhombus rigid link mechanism in Fig. 1(a) the mechanical advantage, MA, may be defined as:

$$
\mathrm{MA}=\frac{v_{\text {in }}}{v_{\text {out }}}=\frac{r_{\text {in }}}{r_{\text {out }}} \frac{((1,5)-(3,5))}{((1,3)-(3,5))},
$$

where $r_{\text {in }}$ is the rigid body mechanism input radius, $r_{\text {out }}$ is the mechanism output radius, $(\cdot, \cdot)$ represents the instant center between two links, and $((\cdot, \cdot)-(\cdot, \cdot))$ represents the distance between two respective instant centers. This equation implies that a mechanism topology that exhibits a large mechanical advantage will have a reduced output speed, $v_{\text {out }}$, for a given input speed, $v_{\text {in }}$.

The above analysis assumes power conservation; however, power is not completely conserved in a compliant mechanism since, when loaded, elastic energy is stored due to member flexibility. Nonetheless, this approach provides the designer an efficient, basic tool (based on geometry alone) for initial selection and modification of structural topologies that have desirable amplification properties. For example, it is observed from Fig. 1 and Eq. (2) that the MA of the mechanism may be modified simply by adjusting the aspect ratio or shape of the rhombus.

\section{II.B. Unit Cell Computational Analysis}

The spectral finite element analysis (SFEA) method ${ }^{11}$ is exploited in the reduced order computational modeling of the various structures presented herein. A detailed description of the analysis of a compliant mechanism unit cell is provided ${ }^{6}$ by the authors' in previous work. Briefly, the unit cell is excited by a pressure wave which is assumed to act normally incident to the bottom layer. Symmetry boundary conditions are used, where rotation degrees of freedom (dof) of all nodes on the left and right edges of the unit cell are constrained while translation dof are left unconstrained. Structural dynamic response is quantified by computing the top layer RMS normal (i.e. transverse) velocity. ${ }^{12}$ Also, to further define performance three compliant mechanism unit cell computational analysis and design tools ${ }^{6}$ are utilized including: 1) a wavenumber-frequency analysis, 2) a frequency response function (FRF) amplitude and phase analysis, and 3) a deformed shape and energy distribution analysis.

\section{Concept for Shape Adaptive Unit Cell}

In this section a novel concept for a shape adaptive unit cell is provided with the intent being to tune structural dynamic response. The rhombus compliant mechanism unit cell topology affords the benefit of easy shape modification to gain a greater understanding of the role of mechanical advantage as it pertains to the top layer RMS normal velocity response of the unit cell. Possible locations for integral actuators are represented by asterisks in the shape adaptive unit cell concept shown in Fig. 2.

Observe that adjusting the aspect ratio of the rhombus by moving the location of the top slider, denoted by link 6 in Fig. 1(a), modifies the location of instant center $(1,5)$. In this manner, the mechanical advantage calculated using Eq. (2) may be decreased. In this study 17 different rhombus compliant mechanism unit cells were considered with MA values ranging from 17 to 1 . However, for the sake of brevity, only the unit cells with a MA of 17, 9, and 1 are shown in Figs. 2(a), (b), and (c), respectively. The shape modifications shown here highlight possibilities for the eventual integration of novel actuators ${ }^{13}$ into unique unit cell designs.

\section{Unit Cell Results}

Each unit cell is assumed to be made of aluminum material with a Young's modulus, $E=7.1 \times 10^{10} \mathrm{~N} / \mathrm{m}^{2}$, and density, $\rho=2700 \mathrm{~kg} / \mathrm{m}^{3}$. A complex modulus, $E^{*}=E(1+\mathrm{i} \eta)$, is used in all computations with a loss factor, $\eta=0.01 .{ }^{12}$ For simplicity, the regions where actuators reside within each unit cell are assumed to have the same material parameters while recognizing that real actuators will possess different mass and stiffness properties. 


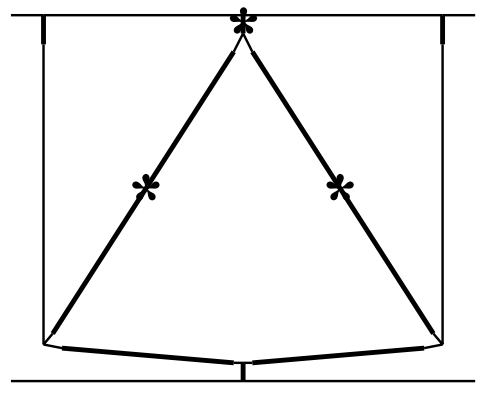

(a)

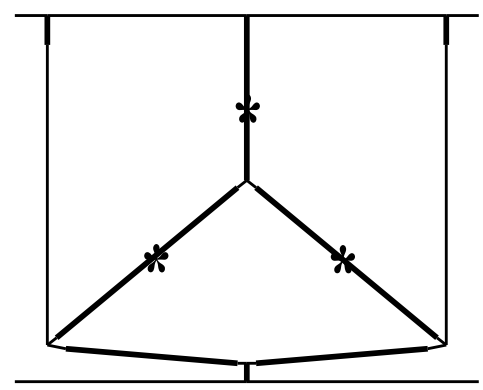

(b)

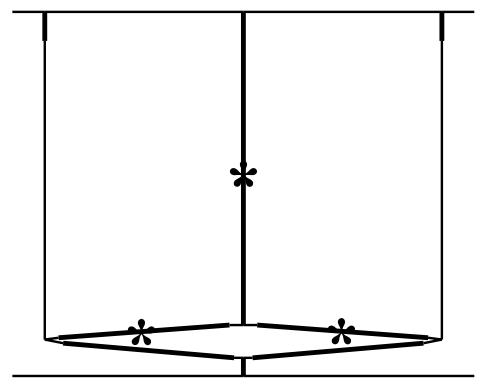

(c)

Figure 2. Concept for shape adaptive unit cell (theoretical location of integral actuators denoted by asterisks): (a) $\mathrm{MA}=17$; (b) $\mathrm{MA}=9$; (c) $\mathrm{MA}=1$.

The unit cell dimensions in Fig. 1(b) are taken as $0.1 \mathrm{~m}$ by $0.1 \mathrm{~m}$. This sizing allows for possible MA values ranging from 17 to 1 . In Fig. 1(b) the core elements of the compliant mechanism unit cell that are 2.5 $\mathrm{mm}$ wide are shown as thinner lines. Thicker lines in Fig 1(b) represent stiffer members that are assigned a width of $5.0 \mathrm{~mm}$; refer to the discussion by Dede and Hulbert. ${ }^{7}$ The incident pressure field amplitude applied to each unit cell is $p_{\text {in }}=1 \mathrm{~N} / \mathrm{m}^{2}$. Top layer RMS transverse velocity is computed using a standard reference velocity, $\mathrm{v}_{\text {ref }}=10^{-8} \mathrm{~m} / \mathrm{s}$.

Sample results for the three unit cells with respective MA values of 17, 9, and 1 are highlighted in Fig. 3, where the top layer RMS transverse velocity response for each unit cell was computed over the $1-10 \mathrm{kHz}$ mid-frequency range using $10 \mathrm{~Hz}$ step increments. The response of the unit cell with $\mathrm{MA}=17$ is established as the baseline for comparison. Above $1 \mathrm{kHz}$ and prior to the beginning of the first attenuation zone, three resonances of this unit cell are evident at 1470, 1840, and $2070 \mathrm{~Hz}$. The first attenuation zone exists in the frequency range of $2320-4000 \mathrm{~Hz}$ with the first cutoff frequency at $4080 \mathrm{~Hz}$. Additional reduction in RMS normal velocity response is present between 4500 and $5500 \mathrm{~Hz}$. Increased modal density occurs from 5500 to $7500 \mathrm{~Hz}$ which leads up to the second attenuation zone centered around $9240 \mathrm{~Hz}$.

Observe in Fig. 3 that as the unit cell mechanical advantage is reduced from 17 to 9, and then to 1 , RMS normal velocity response changes. The breadth of the first attenuation zone is reduced from $2320-$ $4000(\mathrm{MA}=17)$ down to $2320-3500 \mathrm{~Hz}(\mathrm{MA}=1)$. Additionally, the respective average RMS normal velocity response within each frequency range noticeably increases from 21.0 to $40.5 \mathrm{~dB}$. The impact of decreasing the unit cell mechanical advantage on the second attenuation zone is less significant. Still, from 7500 to $9240 \mathrm{~Hz}$ a measurable rise of $8.5 \mathrm{~dB}$ exists across results. Also of interest in this figure are the unit cell attenuation zone primary anti-resonances at $2320 \mathrm{~Hz}$ and $9240 \mathrm{~Hz}$. The locations of these features remain unchanged as mechanical advantage is decreased since they are an artifact of the unit cell bottom layer fixed material parameters and the width or sizing of the unit cell, as detailed in Dede and Hulbert. ${ }^{6}$

Beyond the differences above, there are two overall effects of reducing the unit cell mechanical advantage. First, for a given unit cell, it is evident that there is a shifting of resonances (this can be determined by counting the number of peaks for a given unit cell starting from left to right in Fig. 3). This shifting can be attributed to a reduction in the mass of the unit cell; the masses of the unit cells with $\mathrm{MA}=1$ and $\mathrm{MA}=9$ are, respectively, $\sim 96 \%$ and $\sim 95 \%$ of the mass of the unit cell with $\mathrm{MA}=17$. Reduced mass, exclusive of any other changes to the system, leads to a 'stiffer' dynamic response if one broadly assumes the unit cell may be idealized as a simple single dof spring-mass system. ${ }^{14}$ However, this effect is somewhat artificial since the mass of the unit cell should not change if actuators are properly incorporated into the computational model. Instead a redistribution of mass would occur due to the shape change of the integral compliant mechanism. Nonetheless, resonance shifting due to varying mass is a factor in determining the final position of the major peak at $3500 \mathrm{~Hz}$ for the unit cell with $\mathrm{MA}=1$ in Fig. 3.

The second effect of reducing mechanical advantage is the overall increase in the amplitude of vibratory response within both attenuation zones. This behavior is an artifact of the increased transverse displacement of the output of the compliant mechanism unit cell relative to that of the input which is illustrated by computing the FRF from $1-5 \mathrm{kHz}$ for all three unit cells. In Figs. 4(a) and (b) FRF amplitude and phase are scaled by respective factors of 100 and 1/10, while in Fig. 4(c) FRF amplitude and phase are scaled 


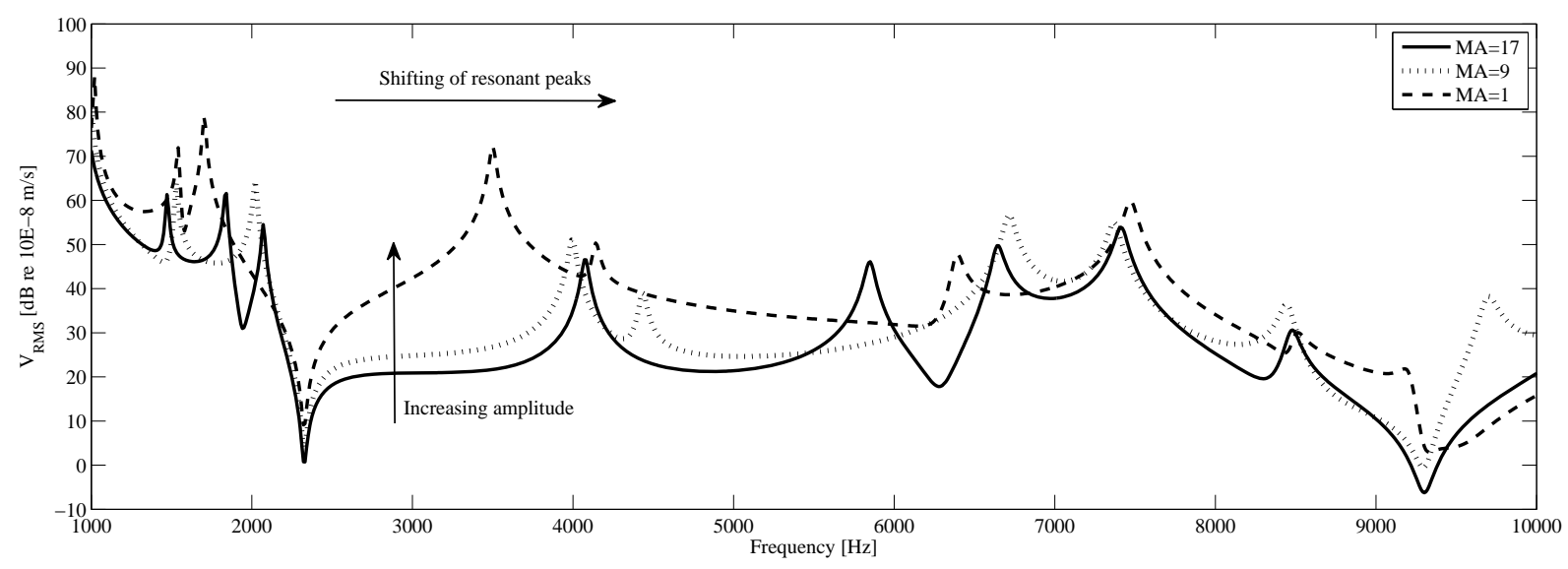

Figure 3. Top layer RMS velocity of compliant mechanism unit cells with MA values of 17, 9, and 1 .

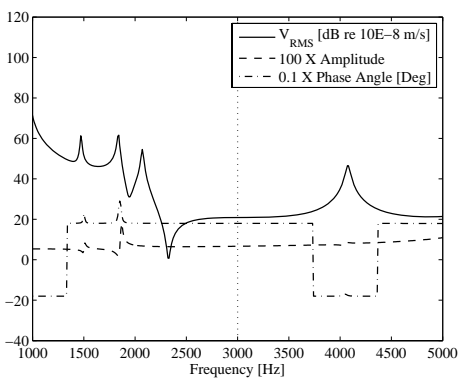

(a)

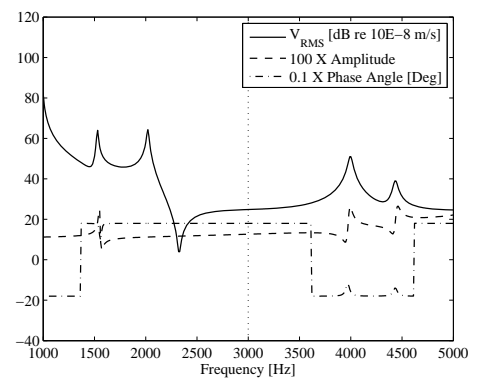

(b)

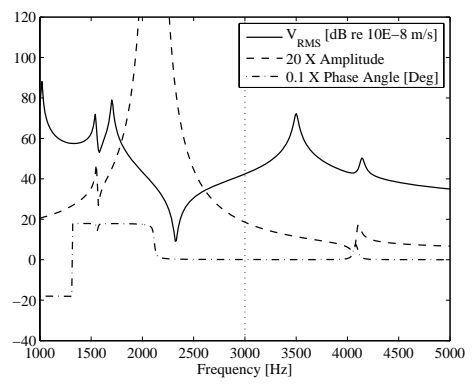

(c)

Figure 4. Rhombus compliant mechanism unit cell FRF (dotted vertical line in each figure denotes the frequency of interest at $3000 \mathrm{~Hz}$ ): (a) $\mathrm{MA}=17$; (b) $\mathrm{MA}=9$; (c) $\mathrm{MA}=1$.

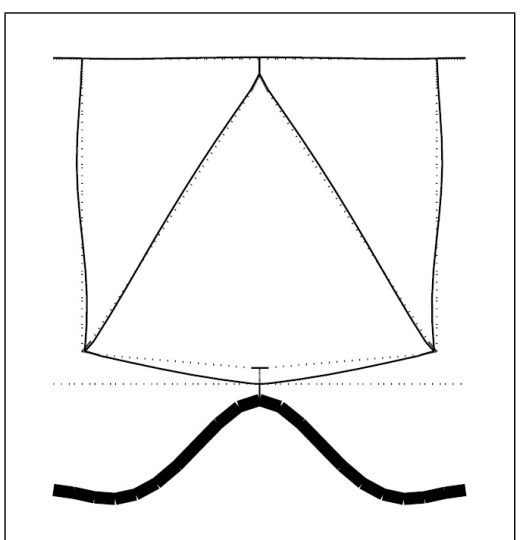

(a)

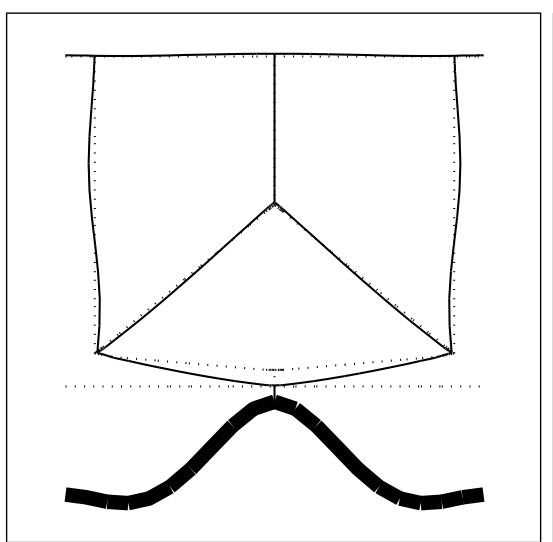

(b)

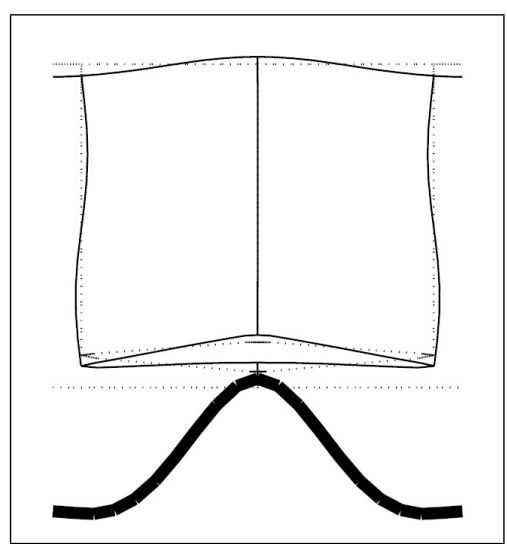

(c)

Figure 5. Deformed shape and energy distribution of compliant mechanism unit cell at $3000 \mathrm{~Hz}$ (continuous lines denote deformed shape; dotted lines denote undeformed shape; line thickness represents energy content): (a) MA=17; (b) $\mathrm{MA}=9$; (c) $\mathrm{MA}=1$. 
by respective factors of 20 and $1 / 10$. Comparing the response at $3000 \mathrm{~Hz}$ across all three figures reveals that amplitude substantially increases as MA decreases. Also, as demonstrated by the phase in Fig. 4(c), response transitions away from the inverter type expected based on the behavior of an equivalent rigid link mechanism.

The deformed shape and energy distribution at $3000 \mathrm{~Hz}$ for all three unit cells provides further insight into these changes in performance. For the unit cell with $\mathrm{MA}=17$, Fig. 5(a), and $\mathrm{MA}=9$, Fig. 5(b), the transverse wave and energy in the bottom layer generates an out-of-phase, 'rigid link mechanism' type motion within the core that is associated with the bending of specific structural members. (Note: line thickness in Fig. 5 represents energy content). In contrast, the unit cell with $M A=1$, Fig. 5(c), exhibits an in-phase, 'nonrigid link mechanism' type behavior, where the transverse motion of the bottom layer is more efficiently transmitted to the unit cell top layer.

\section{Global Structure Results}

Moving to the global view, a comparison is made between two truss-like structures comprising a parallel assemblage of 10 rhombus compliant mechanism unit cells and having respective mechanical advantage values of 17 and 1. Overall dimensions for each structure are $1.0 \mathrm{~m}$ by $0.1 \mathrm{~m}$. In Figs. 6(a) and (b) thicker lines denote members with less compliance. Each structure is assumed to be rigidly connected to ground at its left and right end with all dof constrained for the nodes that reside on either end. It is also assumed that the bottom layer of each structure is subject to a normally incident pressure wave with the same amplitude defined in the unit cell analysis. The top layer RMS normal velocity response of each structure is the engineering quantity of interest, where the established standard reference velocity value is used.

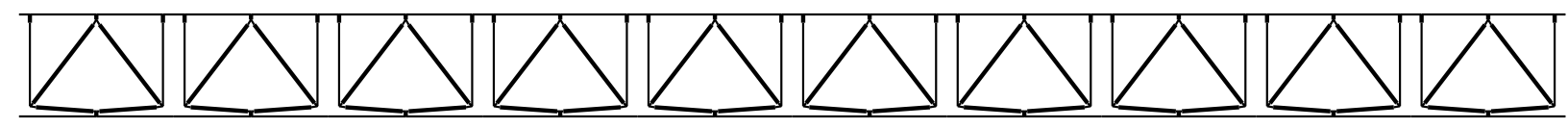

(a)

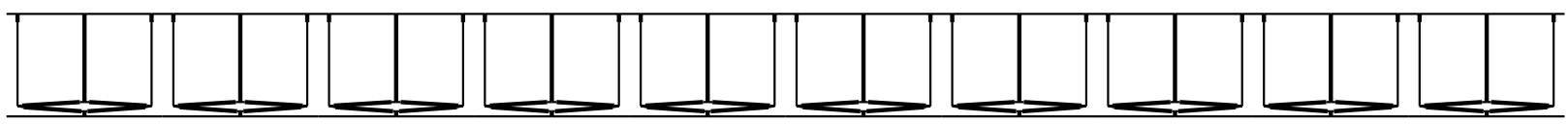

(b)

Figure 6. Compliant mechanism structure composed of 10 unit cells: (a) MA=17; (b) MA=1.

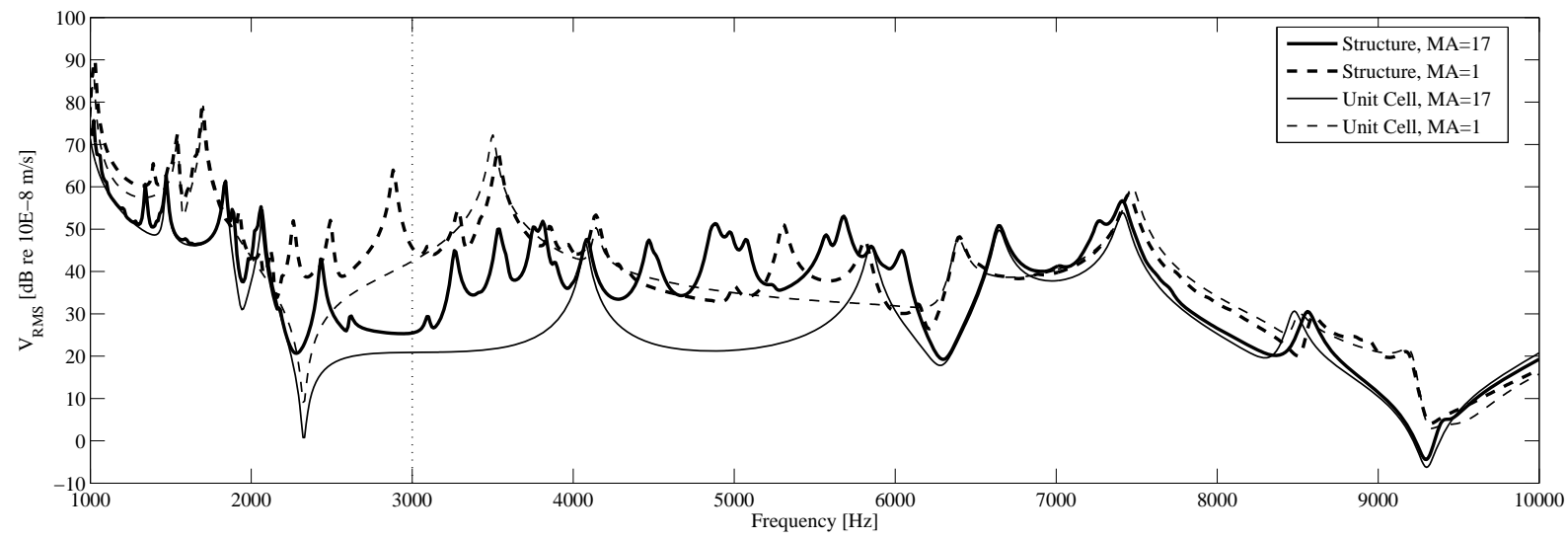

Figure 7. Top layer RMS velocity comparison for rhombus compliant structures and respective unit cells with MA values of 17 and 1 (dotted line denotes the frequency of interest at $3000 \mathrm{~Hz}$ ).

The top layer RMS normal velocity response of each structure is shown in Fig. 7 along with respective unit cell responses. For each structure, increased modal density occurs ${ }^{6,7}$ across the $1-10 \mathrm{kHz}$ frequency range as a result of interaction between the multiple unit cells that are assembled into a global structure. This interaction represents a limitation of the unit cell method since several of the additional resonances 


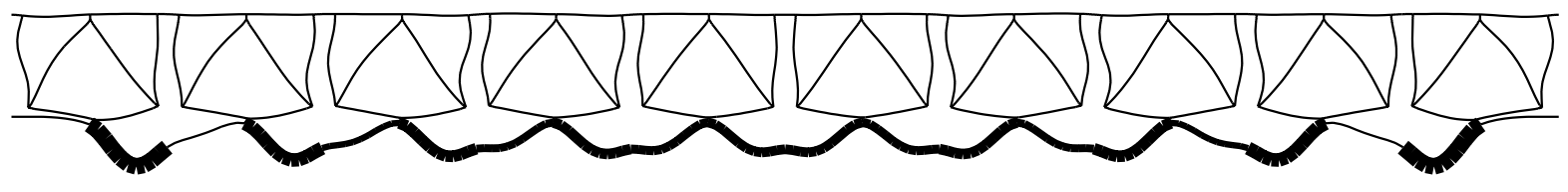

(a)

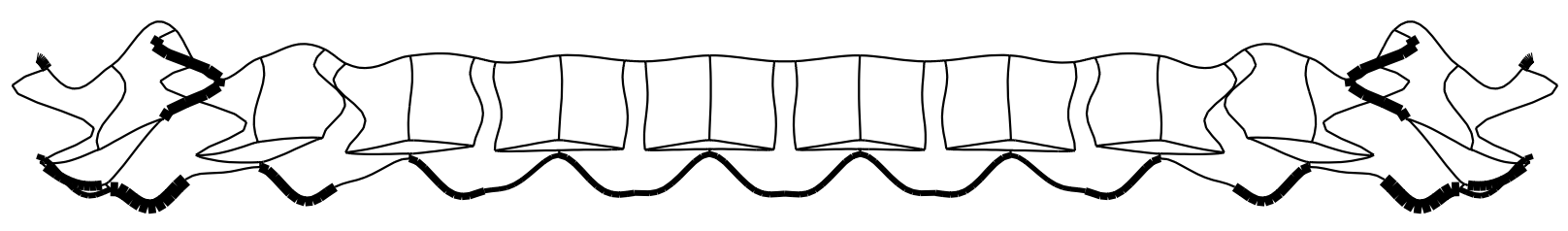

(b)

Figure 8. Deformed shape and energy distribution of rhombus compliant mechanism structure at $3000 \mathrm{~Hz}$ (line thickness represents energy content): (a) $\mathrm{MA}=17$; (b) $\mathrm{MA}=1$.

have significant amplitude. Despite this limitation, local unit cell response is a reasonable predictor of global structural dynamic performance, especially above $5 \mathrm{kHz}$.

Comparing the two global responses reveals how modifying the internal shape of the structure significantly influences the location of resonant peaks and anti-resonant troughs. In terms of amplitude, over the 2320$4000 \mathrm{~Hz}$ frequency range, the structure with $\mathrm{MA}=17$ exhibits an average RMS transverse velocity value of $34.0 \mathrm{~dB}$, which is $14.3 \mathrm{~dB}$ lower than the average value for the structure with $\mathrm{MA}=1$. Likewise, near the second attenuation zone from $7500-9240 \mathrm{~Hz}$, an average reduction of $5.8 \mathrm{~dB}$ is observed.

To further illustrate differences in structural dynamic performance the scaled deformed shape of, and total energy distribution within, each structure was computed at $3000 \mathrm{~Hz}$ and are shown in Fig. 8. The discontinuous nature of portions of the lines in these figures is an artifact of the plot discretization. Note that there is propagation of a transverse wave through the bottom layer of each structure and localization of energy in the bottom layer for unit cells that are adjacent to the fixed ends of each structure. This energy isolation has little influence on the response of the structure with large mechanical advantage, Fig. 8(a), since each unit cell constituent acts as an 'inversor' whose amplification properties prevent the conversion of this localized energy into the transverse motion of the structure top layer. In contrast, the energy localization in the bottom layer of the structure with $\mathrm{MA}=1$, Fig. 8(b), results in large deformations near each fixed end, transmission of this energy into the core of the structure, and greater transverse motion of the structure top layer. Thus, for the sandwich structure with a mechanical advantage of unity, greater coupling of the input side to the output side exists, and each unit cell constituent serves a limited role in the reduction of vibratory response.

\section{Shape Adaptive Composite Unit Cell}

The structural shape modification concept described in Sec. III, and studied in Secs. IV and V, affords substantial control over dynamic response magnitude yet provides the designer with limited ability to shift the primary anti-resonant troughs. In this section the concept for shape adaptive structures is built upon in the development of a multi-material composite unit cell that consists of alternative materials and actuators. Specifically, the integration of adaptive materials into preselected regions of the unit cell enables greater control of anti-resonant behavior.

A multi-material compliant mechanism unit cell concept is proposed where the body of the unit cell is made of acrylonitrile butadiene styrene (ABS), while the bottom layer beams consist of a shape memory alloy (SMA) such as nickel-titanium. A schematic of the unit cell concept is shown in Fig. 9, where actuators are again represented by asterisks and the SMA bottom layer is shown using discontinuous gray lines. Multi-material compliant mechanism prototypes have been developed by Gouker et al., ${ }^{15}$ and their approach shows promise in the development of compliant mechanism structures with embedded active materials and actuators. 


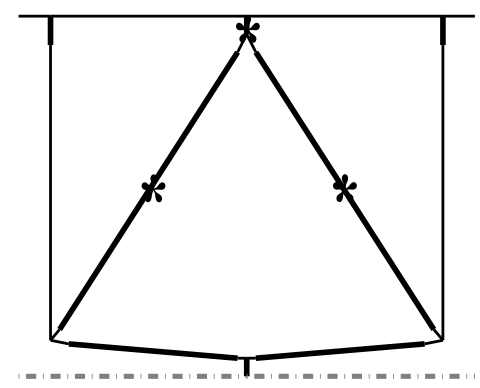

Figure 9. Concept for shape adaptive unit cell (theoretical location of integral actuators denoted by asterisks) with SMA bottom layer (represented by discontinuous gray lines).

The purpose of a unit cell SMA bottom layer is to provide control over material physical parameters. Specifically, by applying a thermal input to the bottom layer the elastic modulus of the SMA material may be increased through a phase transformation from martensite to austenite; refer to Shaw et al. ${ }^{16}$ Increasing the elastic modulus of the bottom layer modifies the location of primary attenuation zone anti-resonances. This conclusion is based on a wavenumber-frequency equation analysis ${ }^{6}$ relating the unit cell anti-resonance values to the bottom layer material parameters and unit cell sizing:

$$
\omega=\left(\frac{2 \pi}{\lambda}\right)^{2} \sqrt{\frac{E I}{\rho A}},
$$

in which $\rho$ is the material density, $E$ is the Young's modulus, $A$ is the cross-sectional area, and $I$ is the area moment of inertia of the bottom layer beam. It is clear from Eq. (3) that for a given unit cell size or wavelength of vibration, $\lambda$, the anti-resonant frequency, $\omega$, is proportional to the square root of the bottom layer material Young's modulus. Therefore, increasing the material Young's modulus raises the unit cell attenuation zone starting frequencies.

Frequency dependent ABS material physical parameters are implemented using the computational model described by Dede and Hulbert. ${ }^{17}$ For conceptual design purposes, the regions within the unit cell where actuators reside are again assumed to have the same material parameters as the bulk of the unit cell body (i.e. ABS). The SMA material parameters assumed for this study are given in Table 1 .

Table 1. SMA material physical parameters ${ }^{16,18}$

\begin{tabular}{cc} 
Material Property & Value \\
\hline Young's modulus, martensite - low temperature & $25.0 \mathrm{GPa}$ \\
Young's modulus, austenite - high temperature & $75.0 \mathrm{GPa}$ \\
Loss factor & 0.035 \\
Density & $6450 \mathrm{~kg} / \mathrm{m}^{3}$
\end{tabular}

The unit cell dimensions are downsized by $50 \%$ to $0.05 \mathrm{~m}$ by $0.05 \mathrm{~m}$ for this analysis. Additionally, the core elements of the compliant mechanism unit cell are reduced in width to $1.25 \mathrm{~mm}$ and $2.5 \mathrm{~mm}$, respectively, for members shown as thin and thick lines in Fig. 9. This downsizing of the unit cell satisfies the goal of keeping the first attenuation zone within the middle frequency (i.e. $1-10 \mathrm{kHz}$ ) range. Specifically, this sizing positions the start of the first attenuation zone at $1820 \mathrm{~Hz}$ when low temperature SMA material parameters are used in Eq. (3). Alternatively, using high temperature SMA material parameters and the aforementioned unit cell sizing produces a first attenuation zone starting frequency of $3140 \mathrm{~Hz}$.

Computational results for this unit cell are shown in Fig. 10 and for clarity are focused only on the first attenuation zone spanning $0-5 \mathrm{kHz}$. Three sets of data are plotted in this figure. The solid line in Fig. 10 represents data for the composite unit cell with $\mathrm{MA}=9$ and the bottom layer SMA material at low temperature. Above $1 \mathrm{kHz}$ there is a resonant peak at $1680 \mathrm{~Hz}$ followed by the beginning of the first attenuation zone at $1820 \mathrm{~Hz}$; this value matches the analytical prediction. The attenuation zone then ends at the cutoff frequency near $2600 \mathrm{~Hz}$ which is followed by another major resonant peak at $4330 \mathrm{~Hz}$. The dotted line in Fig. 10 represents the unit cell with $\mathrm{MA}=17$ and the SMA material again at low temperature. 


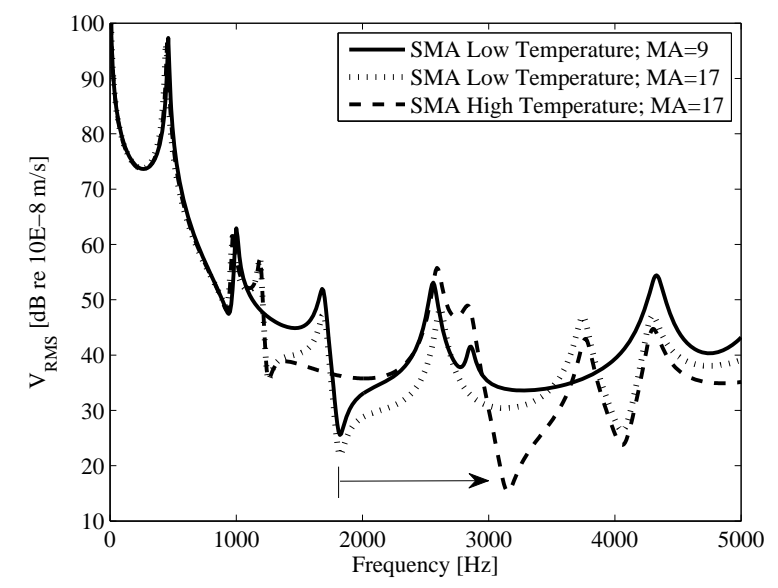

Figure 10. Top layer RMS velocity comparison for composite unit cells containing SMA materials plus actuators.

Increased unit cell mechanical advantage results in almost a $10 \mathrm{~dB}$ reduction of the amplitude of response within the attenuation zone along with some shifting of resonances towards lower frequencies. This resonance shifting is due to the increased mass of the unit cell with $M A=17$ relative to the unit cell with $M A=9$. The dashed line in Fig. 10 is the data for the unit cell with $\mathrm{MA}=17$ and the bottom layer SMA beams at high temperature. Comparing the first (solid) and third (dashed) curves reveals that the start of the first attenuation zone is shifted from $1820 \mathrm{~Hz}$ to $3160 \mathrm{~Hz}$, as expected; see the arrow in Fig. 10. This shifting leads to an $18 \mathrm{~dB}$ decrease in the amplitude of response at $3160 \mathrm{~Hz}$ and a $10 \mathrm{~dB}$ increase in response at 1820 $\mathrm{Hz}$.

Structural dynamic behavior will vary depending on unit cell sizing, amplification properties, and chosen actuator/material properties. Additionally, further work needs to be performed to select actuators and properly incorporate their mass and stiffness into the dynamic computational model of the unit cell and structure. Nonetheless, it is reasonable to conclude that the response in Fig. 10 is representative of combining adaptive materials with actuators within a composite compliant mechanism unit cell. Furthermore, this approach to active control of structural dynamic response should enable tuning of response amplitude and anti-resonances over a broad portion of the mid-frequency range.

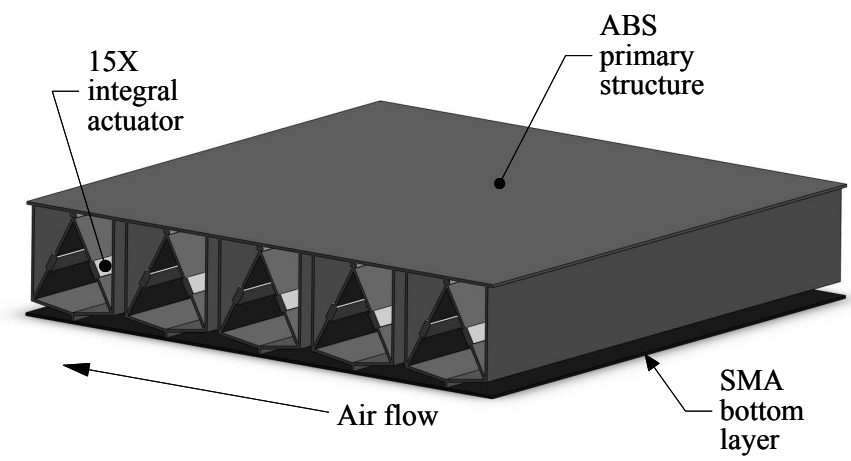

Figure 11. Composite sandwich panel structure with integral compliant mechanisms, actuators, and adaptive materials.

As a focal point for future research, this unit cell can be considered a building block for composite sandwich panels ${ }^{19}$ having integral compliant mechanisms, actuators, and adaptive materials as shown in the conceptual rendering in Fig. 11. Potential applications for this class of structures include composite panels for the reduction of structural-born noise transmitted through an aircraft fuselage or launch vehicle fairing. 


\section{Conclusions}

A new concept for actively tuning the mid-frequency structural dynamic response of periodic structures was presented. A compliant mechanism unit cell was used to study the relationships between shape modification (i.e. amplification properties), adaptive material properties, and response. Results indicate dual effects due to shape modification: 1) shifting of resonant frequencies which is attributable to varying mass; 2) decreased top layer RMS normal velocity response within attenuation zones which is related to an increase in the amplification properties of the unit cell. Results for the composite unit cell with actuators plus adaptive materials indicate that real-time control both over response amplitude and structural anti-resonances is possible. Future work includes incorporating real actuator properties into the computational model to better understand the effects of mass redistribution on structural dynamic response. The analysis of full scale composite sandwich panels with integral compliant mechanisms, actuators, and adaptive materials is a logical next step. Novel potential applications of this method include the design of shape morphing, adaptive material aerospace structures for active control of vibratory performance.

\section{References}

${ }^{1}$ Nashif, A. D., Jones, D. I. G., and Henderson, J. P., Vibration Damping, Wiley, New York, 1985.

2 Johnson, C. D., "Design of Passive Damping Systems," Journal of Mechanical Design, Vol.117 (Issue B), No. 171, 1995, pp. 171-176.

${ }^{3}$ Bhat, W. V., and Wilby, J. F., "Interior Noise Radiated by an Airplane Fuselage Subjected to Turbulent Boundary Layer Excitation and Evaluation of Noise Reduction Treatments," Journal of Sound and Vibration, Vol. 18, No. 4, 1971, pp. 449-464.

${ }^{4}$ Brillouin, L., Wave Propagation in Periodic Structures: Electric Filters and Crystal Lattices, McGraw-Hill, New York, 1946.

${ }^{5}$ Mead, D. J., "Wave Propagation in Continuous Periodic Structures: Research Contributions from Southampton, 19641995," Journal of Sound and Vibration, Vol. 190, No. 3, 1996, pp. 495-524.

${ }^{6}$ Dede, E. M., and Hulbert, G. M., "Analysis, Design, and Optimization of Structures with Integral Compliant Mechanisms for Mid-Frequency Response," International Journal for Numerical Methods in Engineering, Vol. 73, No. 4, 2008 , pp.470-492.

${ }^{7}$ Dede, E. M., and Hulbert, G. M., "Mid-frequency Response of Structures with Integral Compliant Mechanisms: Verification and Validation," Journal of Sound and Vibration, Vol. 313, No. 3-5, 2008, pp. 493-509.

${ }^{8}$ Trease, B. T., "Topology Synthesis of Compliant Systems with Embedded Actuators and Sensors," Mechanical Engineering Dept., Ph.D. Dissertation, Univ. of Michigan, Ann Arbor, MI, 2008.

${ }^{9}$ Larsen, U. D., Sigmund, O., and Bouwstra, S., "Design and Fabrication of Compliant Micromechanisms and Structures with Negative Poisson's Ratio," Journal of Microelectromechanical Systems, Vol. 6, No. 2, 1997, pp. 99-106.

${ }^{10}$ Erdman, A. G., Sandor, G. N., and Kota, S., Mechanism Design: Analysis and Synthesis, Prentice Hall, Upper Saddle River, 2001.

${ }^{11}$ Doyle, J. F., Wave Propagation in Structures: an FFT-based Spectral Analysis Methodology, Springer-Verlag, New York, 1989.

${ }^{12}$ El-Raheb, M., and Wagner, P., "Transmission of Sound Across a Trusslike Periodic Panel; 2-D Analysis," Journal of the Acoustical Society of America, Vol. 102, No. 4, 1997, pp. 2176-2183.

${ }^{13}$ Kornbluh, R., Perline, R., Eckerle, J., and Joseph, J., "Electrostrictive Polymer Artificial Muscle Actuators," Proceedings of the IEEE International Conference on Robotics and Automation, Vol. 3, Leuven, Belgium, 1998, pp 2147-2154.

${ }^{14}$ Den Hartog, J. P., Mechanical Vibrations, McGraw-Hill, New York, 1956.

${ }^{15}$ Gouker, R. M., Gupta, S. K., Bruck, H. A., and Holzschuh, T., "Manufacturing of Multi-Material Compliant Mechanisms using Multi-Material Molding," International Journal of Advanced Manufacturing Technology, Vol. 30, No. 11-12, 2006, pp. 1049-1075.

${ }^{16}$ Shaw, J. A., Chang, B. C., Iadicola, M. A., Leroy, Y. M., "Thermodynamics of a 1-D Shape Memory Alloy: Modeling, Experiments, and Application," Proceedings of the SPIE 10th Annual International Symposium on Smart Structures and Materials, Vol. 2-6, San Diego, CA, 2003.

${ }^{17}$ Dede, E. M., and Hulbert, G. M., "Computational Analysis and Design of Lattice Structures with Integral Compliant Mechanisms," Finite Elements in Analysis and Design, Vol. 44, No. 14, 2008, pp. 819-830.

${ }^{18}$ Zhang, J., Perez, R. J. Lavernia, E. J., "Documentation of Damping Capacity of Metallic, Ceramic, and Metal-Matrix Composite Materials," Journal of Material Science, Vol. 28, No. 9, 1993, pp. 2395-2404.

${ }^{19}$ Dede, E. M., "Analysis, Design, and Optimization of Structures with Integral Compliant Mechanisms for Mid-Frequency Response,” Mechanical Engineering Dept., Ph.D. Dissertation, Univ. of Michigan, Ann Arbor, MI, 2007. 